\title{
The National Diagnostic Reference Level in Routine Digital Radiography Examinations in Iran
}

\author{
B Mohsenzadeh ${ }^{1}$, MR Deevband ${ }^{1 *}$ and R Pouriran ${ }^{2}$ \\ ${ }^{1}$ Department of Medical Physics and Biomedical engineering, Shahid Beheshti University of Medical Sciences and Health Services, Iran \\ ${ }^{2}$ Faculty of Medicine, Shahid Beheshti University of Medical Sciences, Iran
}

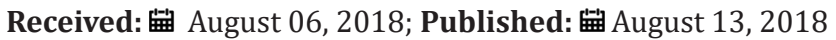

*Corresponding author: MR Deevband, Department of Medical Physics and Biomedical engineering, Faculty of Medicine, Shahid Beheshti University of Medical Sciences and Health Services, Tehran, Iran

\begin{abstract}
Background: The ionizing radiation has an indispensable role in diagnostic radiology and clinical treatments. Apparently, medical exposure in diagnostic radiology pertains to be the preeminent man-made source of radiation.
\end{abstract}

Objective: The nobility of the present scientific study was to establish a precise national diagnostic reference levels (NDRLs) in digital radiography in Iran.

Methods: Conceptually, 15358 patients participated in $85 \mathrm{X}$-ray rooms and the data was obtained from five different age groups: $0>1$ year, $1>5$ years, $5>10$ years, $10>15$ years. And eventually in adults in each projection. The dosimetry protocol was indirect for measuring Entrance Surface Air Kerma (ESAK).

Results: The national diagnostic reference level was settled for Skull (PA), Skull (Lat), Cervical spine (AP), Cervical spine (Lat), Chest (PA), Chest (Lat), Abdomen (AP), Lumbar spine (AP), Lumbar spine (Lat), Pelvis (AP), Thoracic spine (AP), and Thoracic spine (Lat).

Conclusion: A wide variations of radiation dose for x-ray examinations among hospitals in Iran was proceeded; furthermore, it was magnificently attained that the DRL for adult chest (PA) examination could be higher than European countries and Turkey; because of the high exposure parameters could be applied.

Keywords: Digital radiography; Dose Reference Level; Dosimetry in Diagnostic Radiology; Entrance Surface Air Kerma; Radiological Procedures; Digital Radiography; Diagnostic Imaging; Digital Radiography Techniques; Technical Exposure; Dosimeter

Abberevations: PACS: Picture Archiving and Communication System; ICRP: International Commission on Radiological Protection; DRLs: Diagnostic Reference Levels; ESAK: Entrance Skin Air Kerma; ALARA: As Low as Reasonably Achievable; NDRL: National Diagnostic Reference Level; ESDs: Entrance Surface Doses; ESD: Entrance Skin Dose

\section{Introduction}

Diagnostic X-rays has been proposed in medical imaging world-wide. Apparently, it is quite popular and because of the high usage of x-ray among the population, we can see more dosage of radiation than other causes [1]. Ostensibly, patients get exposed to different amount of radiation depending on the type of examination in the field of diagnostic radiology [2]. Transparently, radiological procedures; such as, plain films or digital equipment's, make up to $48 \%$ of the entire diagnostic radiology examinations; in addition, as mentioned above it contributes up to $41 \%$ of the collective dose [3]. Progress in diagnostic imaging has contributed a significant enhance in the health care systems globally; for instance, digital radiography has particularly made the diagnosis easier and faster for physicians [4].

In the past two decades, digital radiography has replaced filmscreen radiography in many medical imaging centers. As a matter of fact, manufacturers produce a wide variety of digital imaging assortment based on different detector and read-out technologies. Apparently, digital detectors enable the performance of full digital picture archiving and communication system (PACS), where images are stored digitally and could be accessible when are needed [5]. The utilization of digital techniques in radiographic imaging are remarkably growing; therefore, it is quite essential to determine the exposure parameters; for instance, the tube voltage could be applied to create images [6] The introduction of digital radiography has been revolutionized from the aspect of dissemination between radiologists and clinicians. Furthermore, it has enhanced the imaging quality promptly and reduced the dosage of use in patients conspicuously [7].

Distantly, albeit digital technique in radiology has made it more accessible for the technicians to reduce the dosage of exposure in 
patients [4]. Additionally, the digital imaging system has a wide dynamic range in which allows overexposure without any particular adverse effect on image quality [5]. It was unambiguous that $80 \%$ of patients who referred to hospitals, at least required a radiographic examination. Evidently, children and infants, constitute $10 \%$ of the total number of X-ray examinees [8]. Translucently, radiation protection in paediatric radiology demands a transspecies attention [9]. considering the higher risk of radiation in children contrary to adults [10]. In order to decrease the rate of radiation in patients, the International Commission on Radiological Protection (ICRP) and the European Commission have advocated the use of diagnostic reference levels (DRLs). Precisely, the DRLs are regulations for assessment of the medical examination performance and could continuously ameliorate the imaging systems well indeed. The continuous improvement of the medical procedure could be attained by monitoring the DRL in certain institution for a given medical devices [11,12].

The Commission of European Communities indicated that the purpose of DRLs is to embolden radiology departments to investigate the radiation dosage in patients peculiarly. Moreover, make national and international comparisons for DRL; nonetheless, DRLs are not universal in nature and radiography practice. Furthermore, the technique varies in countries [13] Limpidly, the ICRP encourages regulatory authorities to set up DRLs which are consistent for the regional, national, or local area in which they would be applied. Contemplate the latest ICRP recommendations and the advances in digital radiology, the essence of patient dose recording and establishment of prognostic reference levels for digital radiology are indicated [14-15].

According to the data, Entrance Skin Air Kerma (ESAK) has been recommended as the pertinent patient dosimetry quantity in simple radiographic examinations because of the following: convenience in measurement, accessible comparison with other studies in different countries or DRLs, and proportionality for effective dosage. Apparently, that is utilized to find the probability of radiation-induced complications [16,17]. Pediatric X-ray examinations are quite more sophisticated and complex in imaging techniques compared to the adult's examinations. Therefore, training the personnel are mandatory [18] Accordingly, considering the "As Low as Reasonably Achievable (ALARA)", it is imperative that radiation doses in a child radiology could be kept as low as possible [19-20] To the best of our knowledge this is the first precise study regarding National Diagnostic Reference Level (NDRL) and a systemic record proposed patient's radiographic data in Iran. Indeed, the purpose of the present study was to establish a comprehensive national plan to specify NDRLs; furthermore, assessed the dose for a random sample in patients including adults and paediatric.

\section{Materials and Methods}

\section{Data Collection Method}

The present study was conducted from January 2014 to January 2016. The probe was performed in all the states in Iran; moreover, a total of 647 digital radiology devices were used. Translucently, 75 hospitals with 85 X-ray rooms were selected across the country; furthermore, 72 state X-ray centers and 13 private medical imaging centers were selected. The selection was done according to the number of patients admitted in the hospitals. As a matter of fact, the 26 hospitals and medical imaging centers were selected in the most populated area in the provinces. The data analysis in this study could be used for establishing national DRL values for digital radiography techniques.

A questionnaire, comprising information on technical exposure settings (including applied tube voltage, tube current, exposure time, and X-ray field size in the detector); the patient data, (sex, age, weight, height, organ thickness, examination type and projection); the institutional data (hospitalization period, room numbers, and number of patients annually); and X-ray machine data (kVpMax, mAsMax, HVL, FSD, output in clinical kVps, production year image receptor type, generator type, grid usage, and exposure setting) were obtained and recorded. Un-ambiguity, the entire data were attained from the trained radiologist technician in the radiology centers. Patients' records were stored in a digital data file to facilitate obtaining entrance surface doses (ESDs).

In the present scientific study, a total of 15358 patients were examined within 2 years. Moreover, patients were categorized into five age groups according to the follows: $0>1$ year, $1>5$ years, $5>10$ years, $10>15$ years, and adults. The standard weight for adult patients was considered $70 \pm 10 \mathrm{~kg}$ approximately; thereby, obese patients with BMI (Body Mass Index) $\geq 30$ were excluded from the survey. The DRL were assessed for each of the following techniques Skull (PA), Skull (Lat), Cervical spine (AP), Cervical spine (Lat), Chest (PA), Chest (Lat), Lumbar spine (AP), Lumbar spine (Lat), Pelvis (AP), Thoracic spine (AP), and Thoracic spine (Lat), and Abdomen (AP).

\section{Evaluation of X-Ray Exposure Parameters in Adult Patients}

One of the most common procedures for evaluating patient diagnostic radiology dosage in diagnostic radiology is measuring the X-ray tube output. The geometry of the X-ray tube output measurement is shown in Figure 1. For patient dose assessment, three steps were followed:

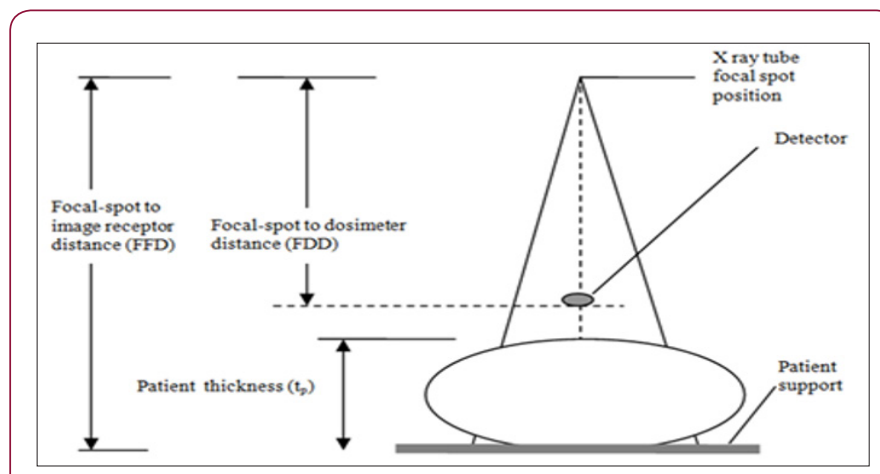

Figure 1: Geometry used for $\mathrm{X}$ ray output measurements.

1) Survey of X-ray exposure parameters in adult and paediatric patients 
2) Measurement of X-ray tube output

3) ESAK Calculation (At least 10 patients were included within the normal Hight and weight in each examination techniques which involved in the study) [21]

\section{Measurements of The X-Ray Tube Output}

The dosimeter (solid state detector) was put on a radiographic table in the central beam axis. The distance of focal spot for detector (FDD) was $100 \mathrm{~cm}$ and field size were $10 \times 10 \mathrm{~cm} 2$ for covering the dosimeter; in order to reduce the effect of scatter radiations to the detector. The $\mathrm{kVp}$ calibration curves have been derived for each $\mathrm{X}$-ray devices by using $\mathrm{kVp}$ ranges from 50-110 in $10 \mathrm{kVp}$ steps and fixed mAS [18]. These curves were analysed for calculatig the X-ray tube output per mAs for different $\mathrm{kVp}$ setting.

\section{Incident Air Kerma and ESAK Calculation}

Primary, for each radiographic examination, incident air kerma was calculated by using the $\mathrm{kVp}$ related output from $\mathrm{kVp}$ calibration curves, applied mAs and correction factor for distances [(FDD/ FSD)2] in each projection. Secondary, the ESAK value was calculated by multiplying incident air kerma in the backscatter factor (BSF) according to the proper field sizes [22].

Entrance surface air kerma $($ ESAK $)=$ Incident air kerma $\times B S F$

\section{Entrance Skin Dose (ESD) Calculation}

Entrance Skin Dose (ESD) was calculated by multiplying ESAK to mass energy absorption coefficients for tissue and air; in which was equal to 1.06 . (this ratio is approximately 1.06 in digital radiology in $110 \mathrm{kvp}$, with $\pm 1 \%$ error] [23].

$$
E S D=E S A K 1.06
$$

\section{DRL (Diagnostic reference level) Calculation}

The DRLs in the present experiment were established by the calculation of ESD values. To calculate DRL firstly, we measured ESD of each radiological technique in medical imaging centre. Then, ESD frequency graph was plotted for each radiology examination, eventually it was obtained that the DRL was in the range of 75 th percentile or roughly third quartile of dose distribution in a sample for its radiology examination deliberately [24].

\section{Data Analysis}

In order to perform statistical analysis, the entire measurements; such as, dosimetry in reference point, measurement of output in the clinical range of kVps, calculation of incident air kerma, and entrance skin air kerma were repeated at least three times to reduce the possibility of errors and anomalous results. Consequently, mean value, error percentage, variation coefficient, standard deviation, and min-max values were calculated by SPSS v16.

\section{Results}

Conspicuously, the distribution of the experimental groups was categorized according to age, sex, mean of weight and height, and the number of patients who participated in each age group which are seen in

Table 1. Distinctly, the number of patients who underwent each $\mathrm{X}$-ray examinations are presented in

Table 2. according to their gender.

Table 3. illustrates the FFD and exposure settings [X-ray tube voltage $(\mathrm{kVp})$, tube current-time product ( $\mathrm{mAs})$, and radiation field size] associated with each X-ray examination.

Table 1: Age group number of patient and patient body characteristics in study (age, height and weight) for each sex.

\begin{tabular}{|c|c|c|c|c|c|c|c|}
\hline \multirow{2}{*}{$\begin{array}{c}\text { Age } \\
\text { Group }\end{array}$} & \multicolumn{2}{|c|}{ weight (kg) } & \multicolumn{2}{|c|}{ height (cm) } & \multirow{2}{*}{ Female } & \multirow{2}{*}{ Male } & \multirow{2}{*}{ Total } \\
\hline & Mean & Range & Mean & Range & & & \\
\hline $\begin{array}{l}0<1 \\
\text { year }\end{array}$ & 7.1 & $\begin{array}{l}2.9- \\
11.3\end{array}$ & 63.8 & $\begin{array}{c}48.6- \\
79\end{array}$ & 468 & 432 & 900 \\
\hline $\begin{array}{l}1>5 \\
\text { year }\end{array}$ & 15.95 & $\begin{array}{l}8.5- \\
23.4\end{array}$ & 93 & $\begin{array}{l}72- \\
114\end{array}$ & 523 & 773 & 1296 \\
\hline $\begin{array}{c}5>10 \\
\text { year }\end{array}$ & 26.25 & $\begin{array}{c}16.5- \\
36\end{array}$ & 126 & $\begin{array}{l}105- \\
147\end{array}$ & 578 & 821 & 1399 \\
\hline $\begin{array}{c}10> \\
15 \\
\text { year }\end{array}$ & 48.75 & $\begin{array}{c}28.5- \\
69\end{array}$ & 153.5 & $\begin{array}{l}135- \\
178\end{array}$ & 749 & 934 & 1683 \\
\hline Adult & 74.7 & $60-80$ & 172.3 & $\begin{array}{l}146- \\
190\end{array}$ & 5040 & 5040 & 10080 \\
\hline Total & & & & & 7358 & 8000 & 15358 \\
\hline
\end{tabular}

Table 2: Distribution of studied group according to sex and examination all exams.

\begin{tabular}{|c|c|c|c|}
\hline X-ray examination & Female & Male & Total \\
\hline Skull (AP/PA) & 924 & 957 & 1881 \\
\hline Skull (LAT) & & & \\
\hline Cervical spine(AP) & 965 & 1023 & 1988 \\
\hline s & & & \\
\hline Chest (PA) & 1083 & 1328 & 2411 \\
\hline Chest (LAT) & 585 & 673 & 1258 \\
\hline Thoracic spine (AP) & 976 & 1032 & 2008 \\
\hline Thoracic spine (LAT) & & & \\
\hline Lumbar spine(AP) & 1123 & 1141 & 2264 \\
\hline Lumbar spine (LAT) & & & 1639 \\
\hline Pelvis (AP) & 797 & 8000 & 15358 \\
\hline Abdomen (AP) & 905 & 1004 & 1909 \\
\hline Total & 7358 & 8003 & \\
\hline
\end{tabular}

Table 3: Summary of the patient attributes and applied exposure parameters.

\begin{tabular}{|c|c|c|c|c|}
\hline X-ray examination & Age & KVp & mAs & FFD (cm) \\
\hline Skull (AP/PA) & $0>1$ year & $48.6(45-55)$ & $14.5(10-20)$ & $90-100$ \\
\hline & $1>5$ year & $55.6(49-59)$ & $19.6(16-25)$ & \\
\hline & $5>10$ year & $58.3(52-60)$ & $21.8(20-25)$ & \\
\hline & $10>15$ year & $63.5(56-69)$ & $25.7(20-32)$ & \\
\hline
\end{tabular}




\begin{tabular}{|c|c|c|c|c|}
\hline & Adult & $68.2(56-77)$ & $28.5(10-32)$ & \\
\hline \multirow[t]{5}{*}{ Skull (LAT) } & $0>1$ year & $46.2(44-56)$ & $11.7(10-20)$ & $90-100$ \\
\hline & $1>5$ year & $52.9(46-56)$ & $16.6(16-25)$ & \\
\hline & $5>10$ year & $55.1(50-58)$ & $18.3(20-25)$ & \\
\hline & $10>15$ year & $61.7(50-63)$ & $21.5(20-32)$ & \\
\hline & Adult & $65.6(51-75)$ & $26.2(10-32)$ & \\
\hline \multirow[t]{5}{*}{ Cervical spine(AP) } & $0>1$ year & $46.7(40-48)$ & $8.3(6-16)$ & $90-100$ \\
\hline & $1>5$ year & $52.3(45-50)$ & $13.9(14-20)$ & \\
\hline & $5>10$ year & $54.7(49-56)$ & $18.2(16-25)$ & \\
\hline & $10>15$ year & $60.1(52-66)$ & $22.1(20-32)$ & \\
\hline & Adult & $65.3(50-75)$ & $26.6(8-32)$ & \\
\hline \multirow[t]{5}{*}{ Cervical spine (LAT) } & $0>1$ year & $46.7(40-48)$ & $8.3(6-16)$ & $90-100$ \\
\hline & $1>5$ year & $54.6(44-53)$ & $15.8(14-20)$ & \\
\hline & $5>10$ year & $55.3(50-60)$ & $18.8(16-25)$ & \\
\hline & $10>15$ year & $61.5(55-71)$ & $22.4(20-32)$ & \\
\hline & Adult & $66.5(58-75)$ & $14.6(8-32)$ & \\
\hline \multirow[t]{5}{*}{ Chest (PA) } & $0>1$ year & $47.8(41-55)$ & $4.1(3.2-8)$ & $80-130$ \\
\hline & $1>5$ year & $56.3(51-63)$ & $6.3(5-10)$ & \\
\hline & $5>10$ year & $63.8(58-71)$ & $7.9(8-14)$ & \\
\hline & $10>15$ year & 72.4(61-79) & $9.8(10-20)$ & \\
\hline & Adult & $80.2(61-135)$ & $12.3(3.2-25)$ & \\
\hline \multirow[t]{5}{*}{ Chest (LAT) } & $0>1$ year & $48.3(48-59)$ & $4.6(3.2-10)$ & $80-130$ \\
\hline & $1>5$ year & $59.8(54-68)$ & $8.1(8-14)$ & \\
\hline & $5>10$ year & $67.6(61-77)$ & $10.5(10-16)$ & \\
\hline & $10>15$ year & $78.7(66-85)$ & $14.2(14-28)$ & \\
\hline & Adult & $87.3(70-135)$ & $17.5(6.4-28)$ & \\
\hline \multirow[t]{5}{*}{ Thoracic spine (AP) } & $0>1$ year & $46.6(42-55)$ & $6.7(3.2-8)$ & $90-100$ \\
\hline & $1>5$ year & $53.3(51-63)$ & $13.5(5-16)$ & \\
\hline & $5>10$ year & $59.5(58-71)$ & $18.2(8-20)$ & \\
\hline & $10>15$ year & $67.4(61-79)$ & $23.6(10-28)$ & \\
\hline & Adult & $74.5(61-86)$ & $25.4(12-40)$ & \\
\hline \multirow[t]{5}{*}{ Thoracic spine (LAT) } & $0>1$ year & $47.6(43-59)$ & $7.9(6.4-10)$ & $90-100$ \\
\hline & $1>5$ year & $57.1(55-68)$ & $15.5(8-18)$ & \\
\hline & $5>10$ year & $65.2(61-79)$ & $19.7(10-25)$ & \\
\hline & $10>15$ year & $76.8(67-87)$ & $25.4(14-32)$ & \\
\hline & Adult & $81(61-90)$ & $31.5(16-51)$ & \\
\hline \multirow[t]{5}{*}{ Lumbar spine(AP) } & $0>1$ year & $48.7(44-55)$ & $13.5(10-18)$ & $90-100$ \\
\hline & $1>5$ year & $56.6(52-65)$ & $19.6(14-32)$ & \\
\hline & $5>10$ year & $62.3(60-69)$ & $23.4(20-38)$ & \\
\hline & $10>15$ year & $69.1(66-75)$ & $30.1(25-50)$ & \\
\hline & Adult & $76(62-96)$ & $35.6(25-64)$ & \\
\hline \multirow[t]{5}{*}{ Lumbar spine (LAT) } & $0>1$ year & $49.5(46-59)$ & $15.3(12-20)$ & $90-100$ \\
\hline & $1>5$ year & $61.3(57-73)$ & $23.6(16-38)$ & \\
\hline & $5>10$ year & $70.4(62-71)$ & $28.2(20-42)$ & \\
\hline & $10>15$ year & $78.3(65-80)$ & $39.8(25-64)$ & \\
\hline & Adult & $85.3(65-100)$ & $45.3(25-80)$ & \\
\hline \multirow[t]{3}{*}{ Pelvis (AP) } & $0>1$ year & $46.1(40-50)$ & $13.3(6.4-14)$ & $90-100$ \\
\hline & $1>5$ year & $52.4(48-61)$ & $17.6(8-20)$ & \\
\hline & $5>10$ year & $57.9(52-65)$ & $19.4(14-32)$ & \\
\hline
\end{tabular}




\begin{tabular}{|c|c|c|c|c|}
\hline & $10>15$ year & $65.7(56-68)$ & $22.8(18-40)$ & \\
\hline & Adult & $73.6(61-85)$ & $26.5(12-45)$ & \\
\hline Abdomen (AP) & $0>1$ year & $48.8(40-50)$ & $13.3(6.4-14)$ & $90-100$ \\
\hline & $1>5$ year & $55.6(48-64)$ & $19.1(8-20)$ & \\
\hline & $5>10$ year & $59.3(52-65)$ & $22.3(14-32)$ & \\
\hline & $10>15$ year & $68.2(56-68)$ & $26.4(18-40)$ & \\
\hline & Adult & $75.7(65-90)$ & $29.7(12-50)$ & \\
\hline
\end{tabular}

The following national diagnostic reference levels were established according to the statistical analysis which was gattered in the present probe.

a) For the age group from 0 to 1 year old: $0.51 \mathrm{mGy}$ for Skull (AP/PA), $0.46 \mathrm{mGy}$ for Skull(Lat), $0.11 \mathrm{mGy}$ for Cervical spine(AP), $0.11 \mathrm{mGy}$ for Cervical spine (Lat), $0.06 \mathrm{mGy}$ for Chest(PA), $0.08 \mathrm{mg}$ Chest (Lat), $0.15 \mathrm{mGy}$ for Thoracic spine (AP), $0.26 \mathrm{mGy}$ for Thoracic spine (Lat), $0.48 \mathrm{mGy}$ for Lumbar spine (AP), $0.74 \mathrm{mGy}$ for Lumbar spine(Lat), and $0.47 \mathrm{mGy}$ for Pelvis (AP) and 0.47 mGy for Abdomen (AP).

b) For the age group from 1 to 5 years old: $0.75 \mathrm{mGy}$ for Skull (AP/PA), $0.68 \mathrm{mGy}$ for Skull(Lat), $0.33 \mathrm{mGy}$ for Cervical spine (AP), $0.38 \mathrm{mGy}$ for Cervical spine (Lat), $0.16 \mathrm{mGy}$ for Chest(PA), $0.23 \mathrm{mGy}$ Chest (Lat), $0.66 \mathrm{mGy}$ for Thoracic spine(AP), 0.74 mGy for Thoracic spine (Lat), $1.06 \mathrm{mGy}$ for Lumbar spine (AP), $1.85 \mathrm{mGy}$ for Lumbar spine (Lat), $0.91 \mathrm{mGy}$ for Pelvis (AP), and 0.98 mGy for Abdomen (AP).

c) For the age group from 5 to 10 years old: $0.93 \mathrm{mGy}$ for Skull (AP/PA), 0.86 mGy for Skull (Lat), $0.53 \mathrm{mGy}$ for Cervical spine (AP), $0.56 \mathrm{mGy}$ for Cervical spine (Lat), $0.38 \mathrm{mGy}$ for Chest(PA), 0.57 mGy Chest (Lat), 1.11 mGy for Thoracic spine(AP), 1.47 mGy for Thoracic spine (Lat), 1.74 mGy for Lumbar spine (AP),
2.87 mGy for Lumbar spine (Lat), 1.23 mGy for Pelvis (AP), and 1.36 mGy for Abdomen (AP).

d) For the age group from 10 to 15 years old: $1.22 \mathrm{mGy}$ for Skull (AP/PA), 1.1 mGy for Skull (Lat), 0.62 mGy for Cervical spine (AP), $0.67 \mathrm{mGy}$ for Cervical spine(Lat), $0.54 \mathrm{mGy}$ for Chest(PA), 0.93 mGy Chest(Lat), $1.48 \mathrm{mGy}$ for Thoracic spine(AP), $2 \mathrm{mGy}$ for Thoracic spine (Lat), 2.41 mGy for Lumbar spine (AP), 3.92 mGy for Lumbar spine (Lat), $1.51 \mathrm{mGy}$ for Pelvis (AP), and 1.86 mGy for Abdomen (AP).

e) For adults: $1.3 \mathrm{mGy}$ for Skull (PA), $1.17 \mathrm{mGy}$ for Skull (Lat), $0.77 \mathrm{mGy}$ for Cervical spine (AP), $0.85 \mathrm{mGy}$ for Cervical spine (Lat), $0.63 \mathrm{mGy}$ for Chest (PA), 1.11 mGy Chest (Lat), 1.73 mGy for Thoracic spine (AP), 2.35 mGy for Thoracic spine (Lat), 2.69 mGy for Lumbar spine (AP), 4.22 mGy for Lumbar spine (Lat), 1.62 mGy for Pelvis (AP), and 2 mGy for Abdomen (AP).

f) (Table 4) presents the summary of minimum, maximum, and the ratio of maximum and minimum, average dose and national diagnostic reference levels (mGy) for each X-ray examinations.

g) The NDRL values for 12 digital radiography examinations in this study is shown in Figure 2. These DRLs were compared with some international DRL in Table 5.

Table 4: A summary of mean, minimum, maximum ESD and NDRL values for all age groups under study irrespective of sex.

\begin{tabular}{|c|c|c|c|c|c|c|}
\hline \multirow[b]{2}{*}{$\mathrm{X}$-ray examination } & \multirow[b]{2}{*}{ Age } & \multicolumn{5}{|c|}{ Entrance surface dose value (mGy) } \\
\hline & & Mean \pm SD & Minimum & Maximum & Ratio Max/Min & $\begin{array}{c}\text { Third quartile in } \\
\text { study }\end{array}$ \\
\hline \multirow{5}{*}{ Skull (AP/PA) } & $0>1$ year & $0.37 \pm 0.41$ & 0.18 & 1.12 & 6.22 & 0.51 \\
\hline & $1>5$ year & $0.58 \pm 0.38$ & 0.21 & 1.37 & 6.52 & 0.75 \\
\hline & $5>10$ year & $0.69 \pm 0.43$ & 0.25 & 1.56 & 6.24 & 0.93 \\
\hline & $10>15$ year & $0.94 \pm 0.42$ & 0.31 & 1.85 & 5.97 & 1.22 \\
\hline & Adult & $0.97 \pm 0.41$ & 0.32 & 2.14 & 6.68 & 1.3 \\
\hline \multirow{5}{*}{ Skull (LAT) } & $0>1$ year & $0.36 \pm 0.39$ & 0.09 & 0.76 & 8.44 & 0.46 \\
\hline & $1>5$ year & $0.51 \pm 0.41$ & 0.22 & 1.29 & 5.86 & 0.68 \\
\hline & $5>10$ year & $0.62 \pm 0.38$ & 0.28 & 1.42 & 5.07 & 0.86 \\
\hline & $10>15$ year & $0.82 \pm 0.40$ & 0.28 & 1.93 & 6.79 & 1.1 \\
\hline & Adult & $0.86 \pm 0.39$ & 0.3 & 2.13 & 7.1 & 1.17 \\
\hline \multirow{5}{*}{ Cervical spine(AP) } & $0>1$ year & $0.09 \pm 0.25$ & 0.08 & 0.56 & 7 & 0.11 \\
\hline & $1>5$ year & $0.27 \pm 0.27$ & 0.09 & 0.74 & 8.22 & 0.33 \\
\hline & $5>10$ year & $0.37 \pm 0.28$ & 0.12 & 1.05 & 8.75 & 0.53 \\
\hline & $10>15$ year & $0.44 \pm 0.23$ & 0.15 & 1.29 & 8.6 & 0.62 \\
\hline & Adult & $0.52 \pm 0.26$ & 0.17 & 1.38 & 8.11 & 0.77 \\
\hline
\end{tabular}

Cite this article: B Mohsenzadeh, MR Deevband, R Pouriran. The National Diagnostic Reference Level in Routine Digital Radiography Examinations in Iran. Biomed J Sci\&Tech Res 7(5)- 2018. BJSTR MS.ID.001578. DOI: 10.26717/ BJSTR.2018.07.001578. 


\begin{tabular}{|c|c|c|c|c|c|c|}
\hline \multirow{5}{*}{$\begin{array}{l}\text { Cervical spine } \\
\text { (LAT) }\end{array}$} & $0>1$ year & $0.09 \pm 0.29$ & 0.08 & 0.53 & 6.63 & 0.11 \\
\hline & $1>5$ year & $0.31 \pm 0.27$ & 0.09 & 0.81 & 9 & 0.38 \\
\hline & $5>10$ year & $0.45 \pm 0.31$ & 0.13 & 1.13 & 8.69 & 0.56 \\
\hline & $10>15$ year & $0.52 \pm 0.28$ & 0.16 & 1.35 & 8.44 & 0.67 \\
\hline & Adult & $0.66 \pm 0.29$ & 0.19 & 1.67 & 8.78 & 0.85 \\
\hline \multirow{5}{*}{ Chest (PA) } & $0>1$ year & $0.06 \pm 0.31$ & 0.02 & 0.15 & 7.5 & 0.06 \\
\hline & $1>5$ year & $0.16 \pm 0.33$ & 0.09 & 0.59 & 6.56 & 0.16 \\
\hline & $5>10$ year & $0.38 \pm 0.29$ & 0.09 & 0.77 & 8.56 & 0.38 \\
\hline & $10>15$ year & $0.54 \pm 0.34$ & 0.11 & 1.08 & 9.82 & 0.54 \\
\hline & Adult & $0.6 \pm 0.31$ & 0.13 & 1.12 & 8.61 & 0.63 \\
\hline \multirow{5}{*}{ Chest (LAT) } & $0>1$ year & $0.07 \pm 0.43$ & 0.03 & 0.21 & 7 & 0.08 \\
\hline & $1>5$ year & $0.18 \pm 0.45$ & 0.07 & 0.62 & 8.86 & 0.23 \\
\hline & $5>10$ year & $0.47 \pm 0.41$ & 0.12 & 1.02 & 8.5 & 0.57 \\
\hline & $10>15$ year & $0.71 \pm 0.42$ & 0.21 & 1.46 & 6.95 & 0.93 \\
\hline & Adult & $0.85 \pm 0.43$ & 0.25 & 1.98 & 7.92 & 1.11 \\
\hline \multirow{5}{*}{$\begin{array}{l}\text { Thoracic spine } \\
\text { (AP) }\end{array}$} & $0>1$ year & $0.13 \pm 0.5$ & 0.08 & 0.61 & 7.63 & 0.15 \\
\hline & $1>5$ year & $0.52 \pm 0.52$ & 0.16 & 1.43 & 8.94 & 0.66 \\
\hline & $5>10$ year & $0.95 \pm 0.48$ & 0.23 & 1.76 & 7.65 & 1.11 \\
\hline & $10>15$ year & $1.23 \pm 0.53$ & 0.29 & 1.8 & 6.21 & 1.48 \\
\hline & Adult & $1.44 \pm 0.5$ & 0.6 & 3.23 & 5.38 & 1.73 \\
\hline \multirow{5}{*}{$\begin{array}{l}\text { Thoracic spine } \\
\text { (LAT) }\end{array}$} & $0>1$ year & $0.21 \pm 0.72$ & 0.08 & 0.7 & 8.75 & 0.26 \\
\hline & $1>5$ year & $0.62 \pm 0.74$ & 0.2 & 1.36 & 6.8 & 0.74 \\
\hline & $5>10$ year & $1.27 \pm 0.7$ & 0.44 & 3.72 & 8.45 & 1.47 \\
\hline & $10>15$ year & $1.7 \pm 0.75$ & 0.47 & 4.08 & 8.68 & 2 \\
\hline & Adult & $2 \pm 0.72$ & 0.64 & 4.72 & 7.35 & 2.35 \\
\hline \multirow{5}{*}{ Lumbar spine(AP) } & $0>1$ year & $0.43 \pm 1.29$ & 0.09 & 0.86 & 9.56 & 0.48 \\
\hline & $1>5$ year & $0.92 \pm 1.27$ & 0.33 & 2.31 & 7 & 1.06 \\
\hline & $5>10$ year & $1.53 \pm 1.32$ & 0.52 & 4.14 & 7.96 & 1.74 \\
\hline & $10>15$ year & $2.14 \pm 1.32$ & 0.69 & 6.48 & 9.39 & 2.41 \\
\hline & Adult & $2.36 \pm 1.29$ & 0.85 & 7.2 & 8.47 & 2.69 \\
\hline \multirow{5}{*}{$\begin{array}{l}\text { Lumbar spine } \\
\text { (LAT) }\end{array}$} & $0>1$ year & $0.63 \pm 1.78$ & 0.25 & 1.75 & 7 & 0.74 \\
\hline & $1>5$ year & $1.6 \pm 1.76$ & 0.63 & 3.98 & 6.32 & 1.85 \\
\hline & $5>10$ year & $2.44 \pm 1.75$ & 0.81 & 5.87 & 7.25 & 2.87 \\
\hline & $10>15$ year & $3.37 \pm 1.8$ & 1.21 & 9.66 & 7.98 & 3.92 \\
\hline & Adult & $3.62 \pm 1.78$ & 1.66 & 10.2 & 6.14 & 4.22 \\
\hline \multirow{5}{*}{ Pelvis (AP) } & $0>1$ year & $0.41 \pm 0.69$ & 0.25 & 1.75 & 7 & 0.47 \\
\hline & $1>5$ year & $0.82 \pm 0.71$ & 0.28 & 2.03 & 7.25 & 0.91 \\
\hline & $5>10$ year & $1.09 \pm 0.68$ & 0.32 & 2.67 & 8.34 & 1.23 \\
\hline & $10>15$ year & $1.35 \pm 0.72$ & 0.48 & 3.58 & 7.46 & 1.51 \\
\hline & Adult & $1.43 \pm 0.69$ & 0.56 & 4.33 & 7.73 & 1.62 \\
\hline \multirow{5}{*}{ Abdomen (AP) } & $0>1$ year & $0.39 \pm 0.79$ & 0.25 & 1.75 & 7 & 0.47 \\
\hline & $1>5$ year & $0.81 \pm 0.77$ & 0.28 & 2.33 & 8.32 & 0.98 \\
\hline & $5>10$ year & $1.12 \pm 0.78$ & 0.3 & 2.21 & 7.37 & 1.36 \\
\hline & $10>15$ year & $1.53 \pm 0.81$ & 0.51 & 3.7 & 7.25 & 1.86 \\
\hline & Adult & $1.65 \pm 0.79$ & 0.58 & 4.04 & 6.96 & 2 \\
\hline
\end{tabular}


Table 5: NDRL values of this study in compare with some international reference dose for 12 digital radiography examinations.

\begin{tabular}{|c|c|c|c|c|c|c|c|c|}
\hline \multirow{2}{*}{$\begin{array}{c}\text { X-ray } \\
\text { examination }\end{array}$} & \multirow[b]{2}{*}{ Age } & \multirow[b]{2}{*}{ This study } & \multicolumn{6}{|c|}{ Third quartile value of ESD (mGy) } \\
\hline & & & $\begin{array}{c}\text { Bushra et al. } \\
\text { (28) }\end{array}$ & $\begin{array}{l}\text { Nigeria } \\
(2004)\end{array}$ & $\begin{array}{l}\text { European } \\
\text { Commission }\end{array}$ & IRISH NDRL & Turkey & UK NDRL \\
\hline \multirow{5}{*}{ Skull (AP/PA) } & $0>1$ year & 0.51 & 0.15 & 0.89 & - & - & - & - \\
\hline & $1>5$ year & 0.75 & 0.42 & 1.21 & 1.5 & - & - & 1.37 \\
\hline & $5>10$ year & 0.93 & 0.48 & 3.26 & - & - & - & - \\
\hline & $10>15$ year & 1.22 & - & - & - & - & - & - \\
\hline & Adult & 1.3 & - & - & 5 & - & - & - \\
\hline \multirow{5}{*}{ Skull (LAT) } & $0>1$ year & 0.46 & - & - & - & - & - & - \\
\hline & $1>5$ year & 0.68 & - & - & 1 & - & - & 0.82 \\
\hline & $5>10$ year & 0.86 & - & - & - & - & - & - \\
\hline & $10>15$ year & 1.1 & - & - & - & - & - & - \\
\hline & Adult & 1.17 & - & - & 3 & - & - & - \\
\hline \multirow{5}{*}{$\begin{array}{l}\text { Cervical } \\
\text { spine(AP) }\end{array}$} & $0>1$ year & 0.11 & - & - & - & - & - & - \\
\hline & $1>5$ year & 0.33 & - & - & - & - & - & - \\
\hline & $5>10$ year & 0.53 & - & - & - & - & - & - \\
\hline & $10>15$ year & 0.62 & - & - & - & - & - & - \\
\hline & Adult & 0.77 & - & - & - & - & - & 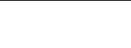 \\
\hline \multirow{5}{*}{$\begin{array}{l}\text { Cervical spine } \\
\text { (LAT) }\end{array}$} & $0>1$ year & 0.11 & - & - & - & - & - & - \\
\hline & $1>5$ year & 0.38 & - & - & - & - & - & - \\
\hline & $5>10$ year & 0.56 & - & - & - & - & - & - \\
\hline & $10>15$ year & 0.67 & - & - & - & - & & - \\
\hline & Adult & 0.85 & - & - & - & - & - & - \\
\hline \multirow{5}{*}{ Chest (PA) } & $0>1$ year & 0.06 & 0.12 & - & - & 0.05 & - & 0.09 \\
\hline & $1>5$ year & 0.16 & 0.18 & 0.52 & 0.1 & 0.053 & - & 0.15 \\
\hline & $5>10$ year & 0.38 & 0.18 & - & - & 0.066 & - & - \\
\hline & $10>15$ year & 0.54 & - & - & - & 0.088 & - & 0.1 \\
\hline & Adult & 0.63 & - & - & 0.3 & - & 0.346 & - \\
\hline \multirow{5}{*}{ Chest (LAT) } & $0>1$ year & 0.08 & - & - & - & - & - & - \\
\hline & $1>5$ year & 0.23 & - & - & 0.2 & - & - & - \\
\hline & $5>10$ year & 0.57 & - & - & - & - & - & - \\
\hline & $10>15$ year & 0.93 & - & - & - & - & - & - \\
\hline & Adult & 1.11 & - & - & 1.5 & - & - & - \\
\hline \multirow{5}{*}{$\begin{array}{l}\text { Thoracic spine } \\
\text { (AP) }\end{array}$} & $0>1$ year & 0.15 & - & - & - & - & - & - \\
\hline & $1>5$ year & 0.66 & - & - & - & - & - & - \\
\hline & $5>10$ year & 1.11 & - & - & - & - & - & - \\
\hline & $10>15$ year & 1.48 & 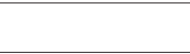 & - & - & - & - & - \\
\hline & Adult & 1.73 & & & & & - & \\
\hline \multirow{5}{*}{$\begin{array}{l}\text { Thoracic spine } \\
\text { (LAT) }\end{array}$} & $0>1$ year & 0.26 & - & - & - & - & - & - \\
\hline & $1>5$ year & 0.74 & - & - & - & - & - & - \\
\hline & $5>10$ year & 1.47 & - & - & - & - & - & - \\
\hline & $10>15$ year & 2 & - & - & - & - & - & - \\
\hline & Adult & 2.35 & - & - & - & - & - & - \\
\hline \multirow{5}{*}{$\begin{array}{l}\text { Lumbar } \\
\text { spine(AP) }\end{array}$} & $0>1$ year & 0.48 & - & - & - & - & - & - \\
\hline & $1>5$ year & 1.06 & - & - & - & - & - & - \\
\hline & $5>10$ year & 1.74 & - & - & - & - & - & - \\
\hline & $10>15$ year & 2.41 & - & - & - & - & - & - \\
\hline & Adult & 2.69 & & & 10 & & - & \\
\hline
\end{tabular}




\begin{tabular}{|c|c|c|c|c|c|c|c|c|}
\hline \multirow{5}{*}{$\begin{array}{l}\text { Lumbar spine } \\
\text { (LAT) }\end{array}$} & $0>1$ year & 0.74 & - & - & - & - & - & - \\
\hline & $1>5$ year & 1.85 & - & - & - & - & - & - \\
\hline & $5>10$ year & 2.87 & - & - & - & - & - & - \\
\hline & $10>15$ year & 3.92 & - & - & - & - & - & - \\
\hline & Adult & 4.22 & - & - & 30 & - & - & \\
\hline \multirow{5}{*}{ Pelvis (AP) } & $0>1$ year & 0.47 & - & - & 0.64 & 0.265 & - & - \\
\hline & $1>5$ year & 0.91 & - & - & 0.92 & 0.475 & - & - \\
\hline & $5>10$ year & 1.23 & - & - & - & 0.8 & - & - \\
\hline & $10>15$ year & 1.51 & - & - & - & 0.89 & - & 1.32 \\
\hline & Adult & 1.62 & - & - & 10 & - & - & - \\
\hline \multirow{5}{*}{$\begin{array}{l}\text { Abdomen } \\
\text { (AP) }\end{array}$} & $0>1$ year & 0.47 & 0.34 & 0.44 & 0.7 & 0.33 & - & - \\
\hline & $1>5$ year & 0.98 & 0.4 & 3.75 & 1 & 0.75 & - & 0.7 \\
\hline & $5>10$ year & 1.36 & 0.45 & 5 & - & - & - & 2.6 \\
\hline & $10>15$ year & 1.86 & - & - & - & - & - & - \\
\hline & Adult & 2 & - & - & 10 & - & 1.948 & - \\
\hline
\end{tabular}

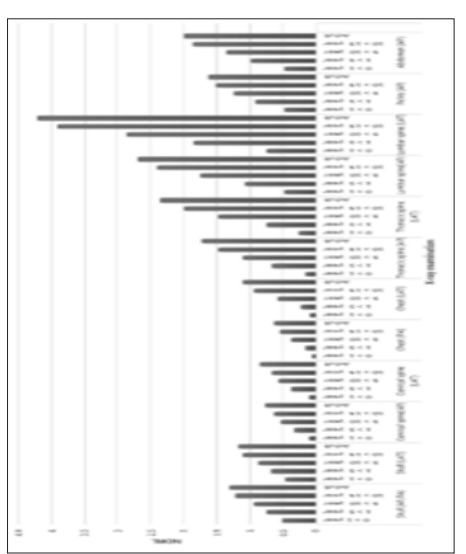

Figure 2: National Diagnostic Reference Level (NDRL) values for 12 digital radiography examinations.

\section{Discussion}

The present scientific study is the first comprehensive national plan which was designed with the cooperation of national authorities, to determine NDRLs in digital X-ray examinations in Iran. Eloquently, the purpose was to examine a vast variety of patients in X-ray imaging centers across the country in the experiment. This survey reveals a significant variation in radiological practice. Ostensibly, the exposure parameters which are set by radiologic technicians could change in a wider rage; for example, in adults the tube loading (mAs) varies from 3.2 to 25; for chest (PA) examinations from 25 to 80 . for lumbar Lat examinations. Substantial variations were recorded in FFD for the same procedures distinctly.

For example, FFD has changed from 90 to $130 \mathrm{~cm}$ for chest (PA) projection and the other examinations demonstrated similar significant variations as well. These considerable variations in exposure parameters led to great differences in mean values of ESAK for the same procedures; up to a factor of 7.65 in hospitals (Pelvis AP). The maximum and minimum ratio in chest PA and Pelvis AP procedures showed extremely large differences of 8.46 and 9, respectively. The outcomes show that the dosage received for the age group $1>5$ was significantly elevated, because of the precise increase in the exposure parameters, particularly mAs. Furthermore, it was obtained that the highest value for DRL was related to Lumbar spine (LAT). Since in over weight patients, the exposure parameters had to be increased; furthermore, due to the sensitivity of children to the radiation than adults this technique should be considered cautiously.

The DRL for adult chest (PA) examination is higher than European and Turkish, because of the high exposure parameters; for instance, high mAs was applied in the radiology centers which contributed in this study compared to the aforementioned countries. The other reason could be that there was not appropriate FFD (120-140cm) selection criteria within the centers indeed, dose difference between various centers due to the used of different conditions and radiographic methods. In the present probe, it was obtained that the significant variations in the ESD values. Based on the obtained data from medical centre examined in this study, it could be pertained that these variations were because of the differences in actual radiation energy and flux, scattering from quite large fields, type of detector, performance of equipment's and processors, filtration, setup from one hospital to another for the same examination. Moreover, because of the employed exposure settings such as $\mathrm{kVp}$, mAs and field size, use of grids and skills of the staff.

The X-ray imaging centers that radiology devices are equipped with automatic exposure control AEC; moreover, the ESD was significantly reduced. During radiography, doses given to children from chest X-ray examination were relatively low; however, due to the high frequency of these technique, their optimization was quite important for the radiology practice.

\section{Limitation}

There were some administrative constraints and systematic errors which are listed below

Quite few voluntary hospitals did not fully cooperate in order to provide required information, Digital radiology devices were 
dispersed all over the country, and extensive changes in exposure parameters amongst centers were observed. To resolve these problems, several centers and hospitals were used. Usually, only limited data was measured for paediatric doses, due to the low number of medical examinations for children compared to adults.

\section{Conclusion}

It was ultimately concluded that through optimizing parameters could be possible to preserve image quality while reducing patient dose [19]. Changes in DRL are affected by factors that have an impact on the exposure of patients. It was observed that the numerical value of DRL depends on the equipment in clinical radiology departments; moreover, high variability of the dose is the reason for harmonizing the different techniques in X-ray services and for desirable diagnostic imaging at reasonable doses for establishment of diagnostic reference levels. Paediatric are more prone than adults to radiation exposure. Therefore, dose monitoring during children digital X-ray examinations and the establishment of local DRLs should be considered strongly. In addition, there should be periodical review of the values of the NDRLs to ensure that they remain appropriate and could be used as an optimization tool. Further studies are suggested to evaluate technicians work quality environment.

\section{Funding and Acknowledgment}

The authors would like to send their best appreciations to the Vice chancellor of Research Centre at Shahid Beheshti University of Medical Sciences (Tehran, Iran) for financial support (Grant No. .474).

\section{References}

1. Giczi F, Pellet S, McLean ID, Meghzifene A (2008) Testing of the Implementation of the Code of Practice on Dosimetry in X-ray Diagnostic Radiology-Hungarian Contribution 42(41).

2. Morales J, Jaramillo W, Puerta J, Arrieta A, Moncada L (2012) A comparison of age-dependent entrance skin doses in pediatric chest exams with diagnostic reference levels for the Antioquia region of Colombia. Radioprotection 47(04): 575-582.

3. Aroua A, Bize R, Buchillier Decka I, Vader JP, Valley JF, et al. (2003) $\mathrm{X}$-ray imaging of the chest in Switzerland in 1998: a nationwide survey. European radiology 13(6): 1250-1259.

4. Lehnert T, Naguib NN, Korkusuz H, Bauer RW, Kerl JM, et al. (2011) Image-quality perception as a function of dose in digital radiography. American journal of roentgenology 197(6): 1399-1403.

5. Korner M, Weber CH, Wirth S, Pfeifer KJ, Reiser MF, et al. (2007) Advances in Digital Radiography: Physical Principles and System Overview 1. Radiographics 27(3): 675-686.

6. Paydar R, Takavar A, Kardan M, Babakhani A, Deevband M, et al. (2012) Patient effective dose evaluation for chest X-ray examination in three digital radiography centers. Iran J Radiat Res 10(3-4): 139-43.

7. Schaefer Prokop C, Neitzel U, Venema HW, Uffmann M, Prokop M (2008) Digital chest radiography: an update on modern technology, dose containment and control of image quality. European radiology 18(9): 1818-1830.

8. Sulieman A, Vlychou M, Tsougos I, Theodorou K (2011) Radiation doses to paediatric patients and comforters undergoing chest $X$ rays. Radiation protection dosimetry 147(1-2): 171-175.
9. Suliman I, Elshiekh E (2008) Radiation doses from some common paediatric X-ray examinations in Sudan. Radiation protection dosimetry 132(1): 64-72.

10. Alatts N, Abukhiar A (2013) Radiation doses from chest X-ray examinations for pediatrics in some hospitals of Khartoum State. Sudan Medical Monitor 8(4): 186.

11. Nyathi T, Nethwadzi LC, Mabhengu T, Pule ML, van der Merwe DG (2009) Patient dose audit for patients undergoing six common radiography examinations: Potential dose reference levels. The South African Radiographer 47(2): 9-13.

12. Johnston D, Brennan P (2000) Reference dose levels for patients undergoing common diagnostic X-ray examinations in Irish hospitals. The British journal of radiology 73(868): 396-402.

13. İnal T, Ataç G (2014) Dose audit for patients undergoing two common radiography examinations with digital radiology systems. Diagnostic and Interventional Radiology 20(1): 100-104.

14. Bouaoun A, Ben Omrane L, Hammou A (2015) Radiation doses and risks to neonates undergoing radiographic examinations in intensive care units in Tunisia. International Journal of Cancer Therapy and Oncology 3(4): 1-6.

15. Hosseini Nasab SMB, Shabestani-Monfared A, Deevband MR, Paydar R, Nabahati M (2017) Estimation of Cardiac Ct Angiography Radiation Dose Toward the Establishment of National Diagnostic Reference Level for Ccta In Iran. Radiation protection dosimetry 174(4): 551-557.

16. Rasuli B, Mahmoud Pashazadeh A, Ghorbani M, Juybari RT, Naserpour M (2016) Patient dose measurement in common medical X-ray examinations in Iran. Journal of Applied Clinical Medical Physics 17(1).

17. Najafi M, Deevband MR, Ahmadi M, Kardan MR (2015) Establishment of diagnostic reference levels for common multi-detector computed tomography examinations in Iran. Australasian physical \& engineering sciences in medicine 38(4): 603-609.

18. Khosravi H, Gholamalizadeh Z, Zadeh MH, Lahroodi KA, Rastkhah N, et al. (2011) SU-E-I-79: Patient Doses Reduction in Simple Radiographic Examinations in Busy Radiology Departments. Medical Physics 38(6): 3414.

19. Zoetelief J (2005) Patient dosimetry for X rays used in medical imaging. Journal of the ICRU 5(2): 1-11.

20. Khoshdel Navi D, Shabestani Monfared A, Deevband M, Abdi R, Nabahati M (2016) Local-Reference Patient Dose Evaluation in Conventional Radiography Examinations in Mazandaran, Iran. Journal of Biomedical Physics and Engineering 6(2): 61-70.

21. Party IOPSiMDW (1992) National protocol for patient dose measurements in diagnostic radiology: National Radiological Protection Board.

22. Zoetelief J (2005) Patient dosimetry for X rays used in medical imaging. Journal of the ICRU 5(2): 1-113.

23. Khoshdel Navi D, Shabestani Monfared A, Deevband M, Abdi R, Nabahati M (2015) Local-Reference Patient Dose Evaluation in Conventional Radiography Examinations in Mazandaran, Iran. Journal of Biomedical Physics and Engineering 6(2): 61-70.

24.Verdun FR, Aroua A, Trueb PR, Vock P, Valley JF (2005) Diagnostic and interventional radiology: a strategy to introduce reference dose level taking into account the national practice. Radiation protection dosimetry 14(1-3): 188-191. 


\section{ISSN: 2574-1241}

DOI: $10.26717 / B J S T R .2018 .07 .001578$

MR Deevband. Biomed J Sci \& Tech Res

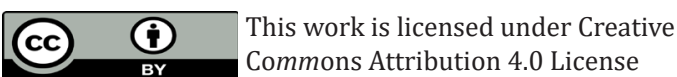

Submission Link: https://biomedres.us/submit-manuscript.php

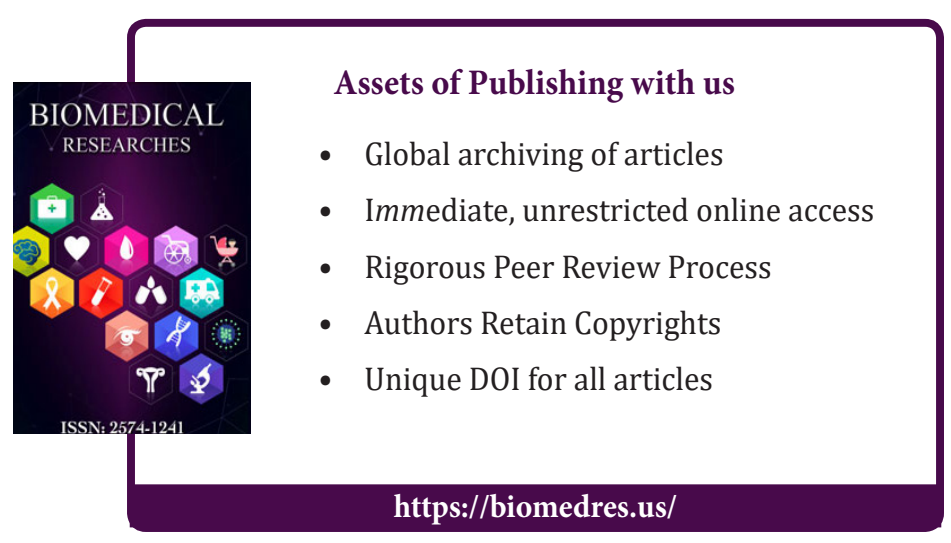

